\title{
Correspondence
}

\section{Section 12 approval: fit for purpose?}

I read this article ${ }^{1}$ with some interest, and with some alarm. Yes, striving for 'evidence-based improvements' in the Section 12 approval/reapproval process is an understandably good thing. However, basing recommendations on a $21.7 \%(5 / 23)$ return rate for a questionnaire is never going to change much behaviour. Not even when this information is 'triangulated... with other sources' are many heads going to be turned.

I believe that those of us who carry out Section 12 assessments in the real world are all too aware of the lack of hospital resources and are thus inclined to seek out every community solution for disposal, given the availability of 'alternative to hospital' teams these days. Particularly when we are considering complex mental illness and mental disorder matters in a social context coupled with a healthy assessment of risk, the decision to detain to hospital for assessment cannot be taken easily or lightly.

Knowing the precise wording of mental health law is important, and we all want to 'do things right'. But in a complex, sometimes heated, community situation we are required to complete the harder additional task of 'doing the right thing', which takes time, thought, experience and some element of wisdom. I am not at all sure that the solutions proposed in this paper will take many in that direction.

Darryl Ballantyne-Watts, Consultant Psychiatrist, Gwent Forensic Psychiatry Service, Aneurin Bevan University Health Board, email: darryl.ballantynewatts@wales.nhs.uk

\section{Declaration of interest}

None.

1 Rigby D, McAlpine L. Section 12 approval: fit for purpose? BJPsych Bull 2019; 43: 251-4.

doi:10.1192/bjb.2020.38

(c) The Author(s) 2020. This is an Open Access article, distributed under the terms of the Creative Commons Attribution licence (http://creativecommons. org/licenses/by/4.0/), which permits unrestricted re-use, distribution, and reproduction in any medium, provided the original work is properly cited.

\section{Section 12 approval: fit for purpose?}

We compliment Rigby and McAlpine on a well-written editorial on Section 12 approval. ${ }^{1}$ The authors raise several pertinent issues about Mental Health Act (MHA) detentions and note that there has been a $47 \%$ increase in the rate of detentions countrywide. They are of the opinion that the increase in detentions is mainly attributable to clinicians not being equipped with the necessary knowledge and training. Rigby and McAlpine suggest more rigorous evidence-based training reinforced by appropriate assessment, including summative assessment using criteria-referenced methods with pass marks determined by the Angoff method. The authors also feel that the approval and revalidation processes need to be more robust.

$\mathrm{We}$, however, are of the opinion that the authors have taken an Occam's razor view by largely attributing the problem to clinicians' training. In our opinion, increases in detention rates are due to multiple factors, and the ' $f$ ix' is not as binary as upgrading training of clinicians or making the approval and revalidation processes more robust. The process of MHA assessment requires two doctors, of which one has to be an independent Section 12 doctor, and an approved mental health professional (AMHP), who is usually (but not invariably) a social worker. All three have to agree to detain a patient with a mental disorder. It is pertinent that the final responsibility for detaining someone under an MHA belongs to the AMHP, who then submits an application to a local hospital for an in-patient bed.

We quote verbatim from the 2018 Care Quality

Commission report ${ }^{2}$ on the use of the MHA to detain people:

'1. The apparent rise in rate of detention since 2010 is in part due to the national data return being more complete.

2. More people are being detained on more than one occasion during a calendar year than was previously the case.

3. Bed numbers have fallen and more people with severe mental health problems are living outside of a hospital setting, and so are at greater risk of being detained.

4. Some people are being detained under the MHA who would previously not have been detained. This is because clinicians are applying the criteria for detention differently to people with certain types of disorder (such as dementia or personality disorder). It could also be because more people with mental health problems are coming to the attention of mental health care workers (for example, through schemes that divert people from the criminal justice system).

5. People who need admission and who would previously have agreed to informal admission are now refusing and are being admitted as detained patients.

6. Admissions (some of which would be formal) that could in the past have been prevented are now not being prevented because less restrictive alternatives in the community are not available.

7. There has been an increase in the total size of the population of England and an increase in the size of those sections of the population that are more at risk of detention.

8. There has been an increase in the prevalence of risk factors for detention, such as social exclusion and problematic, untreated drug and alcohol misuse.'

Glover-Thomas, in a recent review, notes that the availability of mental health beds has decreased, thereby delaying the 'preferred option' of voluntary admission of patients. Therefore, in circumstances when clinicians deem a patient to be in need of care in hospital, resorting to detention 'may be the quickest means of opening up services'. ${ }^{3}$ This factor - in our view - is consequential in 'bumping up' detention rates.

The number of appeals to mental health review tribunals (MHRTs) in England and Wales has risen steadily, from 904 in 
$1980^{4}$ to 31469 in $2014 .^{5}$ This reflects the parallel increase in the number detained. However, the percentage of patients who are successful in obtaining discharge at MHRT hearings is relatively low (only $9 \%$ of all hearings in 2013-2014 resulted in discharge ${ }^{6}$ ), suggesting that patients have been detained appropriately. This, in turn, suggests that training for Section 12 approval is not a factor. However, we agree with Rigby and McAlpine that improvements in training would be beneficial to clinicians in terms of increasing their confidence and knowledge.

Nidhi Gupta, ST5, South Assertive Outreach Team, Birmingham and Solihull Mental Health Foundation Trust, email: nidhigupta@doctors.net.uk; William Calthorpe, Consultant Psychiatrist, South Assertive Outreach Team, Birmingham and Solihull Mental Health Foundation Trust

\section{Declaration of interest}

None.

1 Rigby D, McAlpine L. Section 12 approval: fit for purpose? BJPsych Bull 2019; 43: 251-4.

2 Care Quality Commission. Mental Health Act: The Rise in the Use of the MHA to Detain People in England. CQC, 2018.

3 Glover-Thomas N. Therapeutic jurisprudence and mental health tribunals: changing patterns of demand and its impact. J Soc Welf Fam Law 2019; 41(1): 103-25.

4 Webster L, Dean C, Kessel N. Effect of the 1983 Mental Health Act on the management of psychiatric patients. BMJ 1987; 295: 1529-327.

5 HM Courts \& Tribunals Service. Annual Report and Accounts 2014-15. TSO (The Stationery Office), 2015. Available from: https://assets.publishing. service.gov.uk/government/uploads/system/uploads/attachment_data/ file/433948/hmcts-annual-report-accounts-2014-15.pdf.

6 Care Quality Commission. Monitoring the Mental Health Act in 2013/14. CQC, 2015. Available from: https://www.cqc.org.uk/sites/default/files/ 20150204_monitoring_the_mha_2013-14_report_web.pdf

doi:10.1192/bjb.2020.39
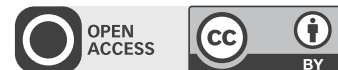

(c) The Authors 2020. This is an Open Access article, distributed under the terms of the Creative Commons Attribution licence (http://creativecommons.org/ licenses/by/4.0/), which permits unrestricted re-use, distribution, and reproduction in any medium, provided the original work is properly cited.

\section{Section 12(2) MHA approval process is fit for purpose}

Rigby and McAlpine have confusingly conflated criticism of Section 12 Mental Health Act (s12 MHA) approval courses, attendance at which is only one of several statutory criteria for s12 approval, with criticism of the overall process of s12 approval. This letter addresses some of the limitations of the article, which in hindsight I'm sure Rigby and McAlpine would prefer to have entitled: 'Are s12 approval courses fit for purpose?'

Before reading further, readers should understand the following.

- A s12-approved doctor is legally defined as 'a medically qualified doctor who has been recognised under section
12(2) of the MHA as having specific expertise in the diagnosis and treatment of mental disorder' and has had training in the application of the MHA. ${ }^{2}$

- The criteria for s12 approval are contained within the statutory instructions ${ }^{3}$ and represent the Government's requirements regarding the work experience, training and qualifications doctors need to possess before they can legally be considered to have the 'specific expertise in the diagnosis and treatment of mental disorder' required to be an s12-approved doctor.

- Regional s12 approval panels have robust governance structures and procedures in place, including audit, that ensure that only those doctors that meet the statutory criteria are approved.

- Doctors require only a basic working knowledge of the MHA to be involved in MHA assessments (MHAAs).

- Doctors do not need to be s12 approved to be involved in MHAAs. If they are not s12 approved then it is preferable that they have personally treated the patient in the past or have some previous knowledge of the patient's case.

- S12 approval courses are not courses on which doctors learn about the MHA, or how to conduct MHAAs, for the first time. The courses serve to reinforce and enhance attendees' knowledge of the MHA and of the MHA Code of Practice. They offer valuable time for discussion among clinicians, with a solicitor present, with debate often focused on the intricacies of the MHA as opposed to the basics.

- Not all s12-approved doctors are actively involved in detaining patients under the MHA. Examples include medical members of the first-tier tribunals (mental health), Second Opinion Appointed Doctors (SOADs) and doctors who produce independent expert reports for court.

Aspects of Rigby and McAlpine's article that need highlighting include the following.

- Rigby and McAlpine imply that s12 doctors may not be 'equipped with the knowledge and skills', particularly knowledge of the MHA, to consider detention of patients under the MHA and that this may have contributed to a rise in detentions over the past decade. There is no evidence to support their view, which in any case has not considered that the decision to apply to detain a person under the MHA does not lie with s12 doctors but with, usually, an approved mental health professional (who makes the application).

- Rigby and McAlpine declare that a lack of formative assessment [in relation to s12 course objectives] is particularly concerning considering that there is evidence to indicate that there are inadequacies in many psychiatrists' understanding of the relevant [MHA] legislation'. Once again, this is an eye-catching assertion for which they offer no convincing evidence. The two papers they cite are more than 20 years old, from 1999 and 1997 respectively, and pre-date the introduction of routine s12 approval courses, which commenced around 2002.

- Rigby and McAlpine state that international applicants with MRCPsych may not be aware of the UK MHA as 'the MRCPsych does not assess UK mental health law'. However, they neglect to mention that s12 legal instructions require that if the applicant has completed all or a substantial part of their training outside England or 